\title{
Atomic data for astrophysics: Fe x soft X-ray lines ${ }^{\star}$
}

\author{
G. Del Zanna ${ }^{1}$, P. J. Storey ${ }^{2}$, N. R. Badnell ${ }^{3}$, and H. E. Mason ${ }^{1}$ \\ 1 DAMTP, Centre for Mathematical Sciences, Wilberforce Road, Cambridge, CB3 0WA, UK \\ e-mail: g.del-zanna@damtp.cam.ac.uk \\ 2 Department of Physics and Astronomy, University College London, Gower Street, London, WC1E 6BT, UK \\ 3 Department of Physics, University of Strathclyde, Glasgow, G4 0NG, UK
}

Received 22 December 2011 / Accepted 14 February 2012

\begin{abstract}
New atomic calculations for Fe $\mathrm{x}$ are presented. They focus on the need to model the soft X-ray spectrum and in particular the line at 94.0 $\AA$ which is the dominant contribution to the Solar Dynamics Observatory (SDO) Atmospheric Imaging Assembly (AIA) $94 \AA$ band in quiet Sun conditions. This line, and others in the band, are due to strong decays from $n=4$ levels. We present new large-scale R-matrix (up to $n=4$ ) and distorted-wave (DW, up to $n=6$ ) scattering calculations for electron collisional excitation and compare them to earlier work. We find significant discrepancies with previous calculations. We show that resonances significantly increase the cross-sections for excitations from the ground state to some $n=4$ levels, in particular to those in the $3 \mathrm{~s}^{2} 3 \mathrm{p}^{4} 4 \mathrm{~s}$ configuration. Cascading from higher levels is also important. We suggest a new identification for the $3 \mathrm{~s}^{3} \mathrm{p}^{6}{ }^{2} \mathrm{~S}_{1 / 2}-3 \mathrm{~s} 3 \mathrm{p}^{5} 4 \mathrm{~s}^{2} \mathrm{P}_{3 / 2}$ transition, that has a predicted intensity larger than the decays from the $3 s^{2} 3 p^{4} 4 s$ levels which were identified by Edlén in 1936 . The results presented here are relevant to our understanding of transitions from $n=4$ levels in a wide range of other ions.
\end{abstract}

Key words. atomic data - line: identification - Sun: corona - techniques: spectroscopic

\section{Introduction}

The soft X-ray (50-170 ̊) spectrum of the quiet and active Sun is rich in $n=4 \rightarrow n=3$ transitions from highly ionised iron ions, from Fe viI to Fe xvi (see, e.g. Fawcett et al. 1968). Very little atomic data are currently available for these ions and the majority of the spectral lines still await firm identification. Soft X-ray spectra of stellar coronae are routinely observed with the Chandra Low Energy Transmission Grating spectrometer (LETG, see Brinkman et al. 2000).

The Solar Dynamic Observatory (SDO) Extreme ultraviolet Variability Experiment (EVE) (Woods et al. 2010) has also been providing soft X-ray spectra. The SDO Atmospheric Imaging Assembly (AIA, see Lemen et al. 2011) has been observing (for the first time routinely) the solar corona in two broad-bands (among others) centred in the soft X-rays, around 94 and $131 \AA$. These SDO/AIA observations provide unprecedented coverage in terms of spatial and temporal resolutions, and can provide new diagnostic applications, once the atomic data are well understood. As shown in O'Dwyer et al. (2010) and Del Zanna et al. (2011), these bands are dominated by Fex and Fe vIII in normal quiet Sun conditions. However, several unidentified spectral lines have been observed in the AIA passbands with high-resolution grazing incidence solar spectrometers (cf. Behring et al. 1976; Manson 1972).

The atomic data for Fe VIII and Fe Ix relevant for the soft X-rays have recently been discussed in O'Dwyer et al. (2012),

\footnotetext{
* The full dataset (energies, transition probabilities and rates) are available in electronic form at our APAP website (www. apap-network.org) as well as at the CDS via anonymous ftp to cdsarc.u-strasbg.fr (130.79.128.5) or via http://cdsarc.u-strasbg.fr/viz-bin/qcat?]/A+A/541/A90
}

where new distorted wave (DW) scattering calculations for these two ions were presented.

This paper focuses on the atomic data for the Fe $\mathrm{x} n=$ $4 \rightarrow n=3$ transitions. One of the main transitions is centred at $94 \AA$ and is expected to provide the dominant signal to the SDO/AIA $94 \AA$ Aand in quiet Sun conditions. Large discrepancies (a factor of six) between predicted and observed count rates in this band have been reported (cf. Aschwanden \& Boerner 2011), which could in part be ascribed to problems in the atomic data for Fe x. Indeed, as shown by Malinovsky et al. (1980), large (at least a factor of two) discrepancies between predicted and observed intensities have been found to be present for all the $3 s^{2} 3 p^{4}$ 4s decays to the ground state.

We set out to resolve these discrepancies with a new set of calculations. We encountered along the way a series of problems and issues with the atomic physics model which turn out to be quite general and very interesting.

These Fe $\mathrm{x}$ transitions are of particular significance for the history of the solar corona. In fact, the famous first identification of a coronal line was given by Edlén (1942) (upon suggestion from Grotrian 1939) as the Fe $\mathrm{x}$ forbidden ${ }^{2} \mathrm{P}_{3 / 2}{ }^{2} \mathrm{P}_{1 / 2}$ transition within the ground configuration. Grotrian's suggestion was based on the pioneering laboratory work by Edlén in the 1930's on the identifications of the soft X-ray lines, in particular the Fe $x 3 s^{2} 3 p^{4} 4 s$ decays to the ground (Edlén 1937).

The paper is organised as follows. In Sect. 2, we give a brief review of previous soft X-ray observations and atomic calculations. In Sect. 3 we outline the methods we adopted for the scattering calculations. In Sect. 4 we present our results and in Sect. 5 we reach our conclusions with respect to $\mathrm{Fe} x$ and other ions. 


\section{Previous observations and atomic data for Fex}

A detailed discussion of the identifications, the historical context, and the atomic data for the $n=2,3$ configurations, giving rise to spectral lines from the EUV to the visible was presented in Del Zanna et al. (2004) and is not repeated here. Del Zanna et al. (2004) presented new identifications and a set of new observed energies that are adopted here. The energies of the $3 s^{2} 3 p^{4} 3 d$ levels have all been confirmed with recent, accurate EUV observations with the Hinode EUV Imaging Spectrometer (Del Zanna 2012).

For the soft X-rays ( $n=4 \rightarrow n=3$ transitions), Edlén carried out the first work on the identification of some $3 s^{2} 3 p^{4} 4 s$ decays to the ground. This was followed by the monumental work by Fawcett et al. (1972), where a number of lines arising from the $3 \mathrm{~s}^{2} 3 \mathrm{p}^{4} 4 l(l=\mathrm{s}, \mathrm{p}, \mathrm{d}, \mathrm{f})$ levels were identified. It is important to keep in mind that only lines with strong oscillator strengths were identified, that some identifications were tentative, and that a large number of lines in the laboratory spectra were left unidentified. Not all of Fawcett's work has been adopted within the NIST compilation.

We have re-analysed some of Fawcett's plates as part of a larger project to sort out the identifications in the Fe soft $\mathrm{X}$-ray spectrum. We have also considered various other experimental sources, in particular the excellent (still the best) grazing-incidence spectra of the full Sun taken in the late 1960s during rocket flights (see Manson 1972; Behring et al. 1972; Malinovsky \& Heroux 1973). Virtually all spectral lines, even at high resolution, are blended. The majority of lines are still unidentified and very few reliable atomic data are available. For these reasons, a full comparison and benchmarking with observations is deferred to a future paper.

The first comprehensive collision strength calculation for Fex was published by Mason (1975). She used the University College London (UCL) distorted-wave DW code (Eissner 1998), which includes the SUPERSTRUCTURE program (Eissner et al. 1974). Only the lowest $3 \mathrm{~s}^{2} 3 \mathrm{p}^{5}, 3 \mathrm{~s} 3 \mathrm{p}^{6}$, and $3 s^{2} 3 p^{4} 3 d$ configurations were included.

Malinovsky et al. (1980) also used the UCL-DW code to calculate collision strengths, but this time for the $n=4$ levels. The authors focused on the $3 s^{2} 3 p^{4} 4 s$ decays to the ground. Large (factors of 5 to 6) discrepancies between the observed (by Malinovsky \& Heroux 1973) and calculated line intensities were found. Cascading from higher levels was found to be important, but difficult to estimate. The inclusion of cascading contributions improved the comparison, but still left discrepancies of a factor of two or more. To our knowledge, no other calculations for the $n=4$ levels have been published since.

For the $n=3$ levels there are a number of calculations. Pelan $\&$ Berrington (2001) published, as part of the Iron Project, a full Breit-Pauli R-matrix calculation for a target including 180 levels arising from the five lowest $n=3$ configurations. Collision strengths were calculated for a total of 7460 energy points in the resonance region (up to 9.95 Ryd). Pelan \& Berrington (2001) only published excitation data from the three levels arising from $3 s^{2} 3 p^{5}$, 3s $3 p^{6}$. Given that there are a number of metastable states for this ion, collision data from those states are also needed (Del Zanna et al. 2004). Hence, a new calculation was needed. In addition, the "top-up" procedure was not applied in Pelan \& Berrington (2001), and collision strengths for the strongest lines were found to be inaccurate (see Aggarwal \& Keenan 2005).

Del Zanna et al. (2004) repeated the earlier Pelan \& Berrington (2001) calculation for the lowest 31 levels due to the $3 s^{2} 3 p^{5}, 3 s 3 p^{6}$, and $3 s^{2} 3 p^{4} 3 d$ configurations but with the addition of high partial wave top-up. These latter data have been used since 2005 within the CHIANTI database (Dere et al. 1997; Landi et al. 2006). Good overall agreement between predicted and observed line intensities using these atomic data was found in Del Zanna et al. (2004) and later in Del Zanna (2012).

Aggarwal \& Keenan (2005) later performed a Dirac Atomic R-matrix Code (DARC) calculation for the lowest 90 levels of the $3 s^{2} 3 p^{5}, 3 s 3 p^{6}$, and $3 s^{2} 3 p^{4} 3 d, 3 s 3 p^{5} 3 d, 3 s^{2} 3 p^{3} 3 d^{2}$ configurations. This calculation was in some respects quite similar to Pelan \& Berrington (2001). However, large differences (up to a factor of two) were found for some allowed transitions such as the 1-3. Relatively good agreement between the Aggarwal \& Keenan (2005) and Del Zanna et al. (2004) is found however, as shown below.

\section{Methods}

The atomic structure calculations were carried out using the AUTOSTRUCTURE program (Badnell 1997) which constructs target wavefunctions using radial wavefunctions calculated in a scaled Thomas-Fermi-Dirac statistical model potential with a set of scaling parameters.

The Breit-Pauli distorted wave calculations were carried out using the recent development of the AUTOSTRUCTURE code, described in detail in Badnell (2011). The continuum distorted waves are calculated using the same form for the distorting potential as specified for the target, but now for the $(N+1)$-electron problem. The electrostatic $(N+1)$-electron interaction Hamiltonian for the collision problem is determined in an unmixed LS-coupling representation. It is then transformed to an LSJ representation. The full $(N+1)$-electron interaction Hamiltonian is transformed to a full Breit-Pauli jK-coupling representation (i.e. including both configuration and fine-structure target mixing) in the same manner as is done for the (innerregion) Breit-Pauli R-matrix method. Collision strengths are calculated at the same set of final scattered energies for all transitions. "Top-up" for the contribution of high partial waves is done using the same Breit-Pauli methods and subroutines implemented in the R-matrix outer-region code STGF. The program also provides radiative rates and infinite energy Born limits. These limits are particularly important for two aspects. First, they allow a consistency check of the collision strengths in the scaled Burgess \& Tully (1992) domain (see also Burgess et al. 1997). Second, they are used in the interpolation of the collision strengths at high energies when calculating the Maxwellian averages.

The R-matrix method used in the scattering calculation is described in Hummer et al. (1993) and Berrington et al. (1995). We performed the calculation in the inner region in $L S$ coupling and included mass and Darwin relativistic energy corrections.

The outer region calculation used the intermediate-coupling frame transformation method (ICFT) described by Griffin et al. (1998), in which the transformation of the multi-channel quantum defect theory unphysical K-matrix to intermediate coupling uses the so-called term-coupling coefficients (TCCs) in conjunction with level energies.

Dipole-allowed transitions were topped-up to infinite partial wave using an intermediate coupling version of the CoulombBethe method as described by Burgess (1974) while non-dipole allowed transitions were topped-up assuming that the collision strengths form a geometric progression in $J$ (see Badnell \& Griffin 2001).

The collision strengths were extended to high energies by interpolation using the appropriate high-energy limits in the 
Table 1. List of a few among the strongest Fe $\mathrm{x}$ lines in the soft X-rays.

\begin{tabular}{lccccccccc}
\hline \hline$i-j$ & Levels & $\begin{array}{c}\text { Int } \\
\text { DW }(n=4)\end{array}$ & $\begin{array}{c}\text { Int } \\
\text { DW }(n=6)\end{array}$ & $\begin{array}{c}\text { Int } \\
\text { RM }\end{array}$ & $\begin{array}{c}\text { Int } \\
\text { RM+ }+ \\
\text { DW }(n=6)\end{array}$ & $\begin{array}{c}\text { Int } \\
\text { CHIANTI }\end{array}$ & $A_{j i}\left(\mathrm{~s}^{-1}\right)$ & $\lambda_{\text {exp }}(\AA)$ & $\lambda_{\text {th }}(\AA)$ \\
& & & & & & \\
\hline $1-202$ & $3 \mathrm{~s}^{2} 3 \mathrm{p}^{5}{ }^{2} \mathrm{P}_{3 / 2}-3 \mathrm{~s}^{2} 3 \mathrm{p}^{4} 4 \mathrm{~s}^{2} \mathrm{D}_{5 / 2}$ & $5.3 \times 10^{-3}$ & $7.5 \times 10^{-3}$ & $9.2 \times 10^{-3}$ & $1.2 \times 10^{-2}$ & $8.9 \times 10^{-3}$ & $3.7 \times 10^{10}$ & 94.012 & 90.46 \\
$3-429$ & $3 \mathrm{~s}^{2} \mathrm{p}^{2} \mathrm{~S}_{1 / 2}-3 \mathrm{~s}^{5} \mathrm{p}^{5} 4 \mathrm{~s}^{2} \mathrm{P}_{3 / 2}$ & $1.7 \times 10^{-2}$ & $1.4 \times 10^{-2}$ & $1.8 \times 10^{-2}$ & $1.8 \times 10^{-2}$ & $5.9 \times 10^{-3}$ & $4.8 \times 10^{10}$ & - & 91.48 \\
$22-267$ & $3 \mathrm{~s}^{2} 3 \mathrm{p}^{4} 3 \mathrm{~d}^{2} \mathrm{G}_{9 / 2}-3 \mathrm{~s}^{2} 3 \mathrm{p}^{4} 4 \mathrm{p}^{2} \mathrm{~F}_{7 / 2}$ & $2.5 \times 10^{-3}$ & $2.8 \times 10^{-3}$ & $3.2 \times 10^{-3}$ & $3.4 \times 10^{-3}$ & $2.8 \times 10^{-3}$ & $1.4 \times 10^{10}$ & 139.869 & 135.95 \\
$1-370$ & $3 \mathrm{~s}^{2} 3 \mathrm{p}^{5}{ }^{2} \mathrm{P}_{3 / 2}-3 \mathrm{~s}^{2} 3 \mathrm{p}^{4} 4 \mathrm{~d}^{2} \mathrm{D}_{5 / 2}$ & $1.1 \times 10^{-3}$ & $1.9 \times 10^{-3}$ & $1.4 \times 10^{-3}$ & $1.6 \times 10^{-3}$ & $6.2 \times 10^{-3}$ & $7.2 \times 10^{10}$ & 77.865 & 75.17 \\
$21-488$ & $3 \mathrm{~s}^{2} 3 \mathrm{p}^{4} 3 \mathrm{~d}^{2} \mathrm{~F}_{5 / 2}-3 \mathrm{~s}^{2} 3 \mathrm{p}^{4} 4 \mathrm{f}^{2} \mathrm{G}_{7 / 2}$ & $2.0 \times 10^{-3}$ & $1.9 \times 10^{-3}$ & $2.2 \times 10^{-3}$ & $2.2 \times 10^{-3}$ & - & $1.6 \times 10^{11}$ & 103.319 & 100.39 \\
$1-30$ & $3 \mathrm{~s}^{2} 3 \mathrm{p}^{5}{ }^{2} \mathrm{P}_{3 / 2}-3 \mathrm{~s}^{2} 3 \mathrm{p}^{4} 3 \mathrm{~d}^{2} \mathrm{D}_{5 / 2}$ & 1.0 & 1.0 & 1.0 & 1.0 & 1.0 & $1.9 \times 10^{11}$ & 174.531 & 163.29 \\
\hline
\end{tabular}

Notes. The relative intensities (photons) $I n t=N_{j} A_{j i} / N_{\mathrm{e}}$ are normalised to the strongest EUV transition (1-30) and were calculated at a coronal electron density of $10^{8} \mathrm{~cm}^{-3}$ and $\log T[\mathrm{~K}]=6.05$. The DW intensities were obtained with the DW calculations. The RM are obtained from the R-matrix calculations, i.e. only including $n=3,4$ levels. The RM+DW is from a combined model where the RM model is augmented by including excitation and radiative data for $n=5,6$ levels from the DW calculation. The last column shows the values calculated with the current CHIANTI model. $A$-values $\left(\mathrm{s}^{-1}\right)$ as obtained from the RM model are shown, as well as experimental $\left(\lambda_{\text {exp }}\right)$ and theoretical $\left(\lambda_{\text {th }}\right.$, from the RM model $)$ wavelengths.

Burgess \& Tully (1992) scaled domain. The high-energy limits were calculated with AUTOSTRUCTURE for both optically-allowed (see Burgess et al. 1997) and non-dipole allowed transitions (see Chidichimo et al. 2003). All the transitions from the ground configuration were visually inspected. General agreement in the background collision strengths was found with the DW values, and at high energies with the limit points.

The temperature-dependent effective collisions strength $\Upsilon(i-j)$ were calculated by assuming a Maxwellian electron distribution and linear integration with the final energy of the colliding electron.

\section{Results}

Several calculations have been performed with different size target expansions and corresponding ion population models have been constructed to predict line intensities and compare with observations. A summary of our investigations is presented here.

\subsection{Initial DW calculations}

We started with various DW calculations systematically increasing the number of configurations up to and including those with $n=6$ valence orbitals. As shown by Del Zanna et al. (2004), a number of metastable levels within the $3 s^{2} 3 p^{4} 3 d$ configuration become significantly populated at coronal densities (up to level 24). Hence, DW excitation rates from the lowest 24 levels have been calculated.

We then performed separate structure calculations for each ion model to calculate all of the radiative data for all transitions among the levels. This ensures that all the cascading from the target configurations is included. We then calculated the level populations and the relative line intensities so as to find out which lines are expected to be strongest in quiet Sun conditions.

Following Malinovsky et al. (1980), the intensities of the soft X-ray lines $(4 s-3 p)$ have been considered relative to the strongest EUV line $(3 \mathrm{~d}-3 \mathrm{p})$. Table 1 shows the details for a few transitions among the strongest lines from the main $n=$ 4 configurations. The relative intensities obtained from two purely DW runs, which are described below, are displayed in the first two intensity columns of Table 1. The first DW ion model includes almost all possible of the $n=3,4$ configurations. The second DW ion model also includes the main $n=5,6$ configurations. The other ion models we built produce similar results. The following two columns show the results obtained with the models described below, while the last one shows the values calculated with the current CHIANTI model, which has the Malinovsky et al. (1980) collisional data.

One of the strongest lines in the soft X-rays is the $3 s^{2} \quad 3 p^{5} \quad{ }^{2} P_{3 / 2}-3 s^{2} 3 p^{4} 4 s^{2} D_{5 / 2}$ identified by Edlén (1937) at $94.012 \AA$. The relative intensity with the $n=4 \mathrm{DW}$ model is $5.3 \times 10^{-3}$ (in photons), i.e. almost six times weaker than observed $\left(3.2 \times 10^{-2}\right)$ by Malinovsky \& Heroux (1973) and about a factor of two lower than calculated by Malinovsky et al. (1980) $\left(1.0 \times 10^{-2}\right)$. As shown by Malinovsky et al. (1980), cascading from higher levels does increase the population of the $4 \mathrm{~s}^{2} \mathrm{D}_{5 / 2}$, but only at the 10-20\% level. Our large $n=6 \mathrm{DW}$ model predicts a relative intensity of $7.5 \times 10^{-3}$, larger as expected, but also lower than the value calculated by Malinovsky et al. (1980) $\left(1.2 \times 10^{-2}\right)$.

Similar discrepancies between Malinovsky et al. (1980) and our results are present for the other lines in the same transition array. The differences between our results and Malinovsky et al. (1980) in the calculated values should not be present since very similar (DW) scattering approximations have been used. Actually, for dipole-allowed lines, Malinovsky et al. (1980) only calculated DW collision strengths at 12 and 20 Ryd, while a semi-classical approximation, based on Burgess (1964), was used at 40 and 80 Ryd. It is not entirely clear which set of configurations was used by Malinovsky et al. (1980). However, we have run a DW calculation including the same set of configurations as listed in their paper, and the differences remain. It turns out that the differences are due to significantly overestimated collision strengths by Malinovsky et al. (1980), as shown below.

The overestimation of collision strengths by Malinovsky et al. (1980) only makes the problem worse in terms of comparison with solar data. The cause could in part be due to an incorrect photometric calibration of the Malinovsky \& Heroux (1973) spectrum in the soft X-rays. A discussion of this is deferred to a future paper. However, even just considering the soft X-rays, significant problems are still present. In particular, the DW calculations clearly indicate that the 3s $3 \mathrm{p}^{6}{ }^{2} \mathrm{~S}_{1 / 2}-3 \mathrm{~s} 3 \mathrm{p}^{5} 4 \mathrm{~s}^{2} \mathrm{P}_{3 / 2}$ transition is almost three times stronger than the $3 \mathrm{~s}^{2} 3 \mathrm{p}^{5}{ }^{2} \mathrm{P}_{3 / 2}-3 \mathrm{~s}^{2} 3 \mathrm{p}^{4} 4 \mathrm{~s}^{2} \mathrm{D}_{5 / 2} 94 \AA$ line. This is caused by a strong forbidden excitation from the ground state to the $3 \mathrm{~s} 3 \mathrm{p}^{5} 4 \mathrm{~s}{ }^{2} \mathrm{P}_{3 / 2}$, with collision strengths much higher than those to the $3 \mathrm{~s}^{2} 3 \mathrm{p}^{4} 4 \mathrm{~s}$ levels, as discussed below. 
The $3 \mathrm{~s} 3 \mathrm{p}^{5} 4 \mathrm{~s}{ }^{2} \mathrm{P}_{3 / 2}$ level then decays via a strong dipoleallowed transition. This transition has not been identified previously. However, our ab-initio calculations predict this line to fall around 95-96 $\AA$. At around these wavelengths there are no lines in the solar spectrum which are two or three times stronger than the $94 \AA$ line. The same holds for the laboratory plates from Fawcett which we have analysed.

All of the DW calculations we have carried out produced similar values. We have also run similar calculations for other iron ions and found the same situation: strong transitions from $3 \mathrm{~s} 3 \mathrm{p}^{q} 4 \mathrm{~s}$, not identified by Edlén or Fawcett. In general, we have found significant numbers of unidentified lines, stronger than those that have been identified, which complicates the benchmarking of the atomic data. As stated previously, a full discussion of benchmarking with solar and laboratory spectra is beyond the scope of this paper and is deferred to a future paper.

The only reasonable solution to the problem is that all of the excitations to the $3 s^{2} 3 p^{4} 4 s$ levels are significantly underestimated by the DW calculations. Indeed, we previously found a similar situation for Fe IX, as discussed in O'Dwyer et al. (2012). We found that there are resonances which increase significantly the collision strengths to the $3 s^{2} 3 p^{5} 4 s$ levels. A purely (nonresonant) DW calculation would underestimate by at least a factor of two the intensities of any decays from these levels, compared to what is obtained with an R-matrix calculation (Storey et al. 2002).

\subsection{Estimate of resonance contribution}

A full R-matrix calculation with $n=4,5$ levels is challenging, so before embarking on such a calculation we performed various DW calculations to estimate which configurations would be likely to be producing resonances in the collision strengths for the spectroscopically important configurations/levels. For each model, we calculated all the collision strengths at threshold between all the levels. The details of two of such calculations are given below. Here, we are using results form the larger of the two calculations.

To assess which configurations would contribute significantly, we considered two steps, the first being dielectronic capture which is directly proportional to the excitation collision strengths. Only levels with similar excitations from the ground configuration can be important. The second step is the Auger decay, which has a rate proportional to the excitation cross-section between the levels (Burgess 1965). If we identify all first step excitations that are stronger or comparable with the direct excitations of interest then we necessarily have all possible candidates for strong resonance contributions. Whether these do in fact contribute strongly, depends on step two.

Consider as an example the $3 s^{2} 3 p^{4} 4 s^{2} D_{5 / 2}$ level which is populated by a dipole excitation from the ground state but with the relatively small threshold collision strength of 0.027 (see Fig. 1). The collision strength for the dipole forbidden excitation of the $3 s^{2} 3 p^{4} 4 p^{2} \mathrm{P}_{3 / 2}$ from the ground state, on the other hand is large, 0.6 at threshold, implying a large dielectronic capture rate to the resonances converging on this state. These resonances can autoionize into all possible open channels but the DW calculations show that the two main routes leave the ion either in its ground state or in the $3 \mathrm{~s}^{2} 3 \mathrm{p}^{4} 4 \mathrm{~s}^{2} \mathrm{D}_{5 / 2}$ level. The threshold collision strengths, 0.6 and 2.1 respectively, provide an estimate of the branching ratio between these routes, with $80 \%$ leaving the ion in the $3 s^{2} 3 p^{4} 4 s{ }^{2} D_{5 / 2}$ level. Since the average effect of the resonances converging on the $3 s^{2} 3 p^{4} 4 p{ }^{2} \mathrm{P}_{3 / 2}$ can be

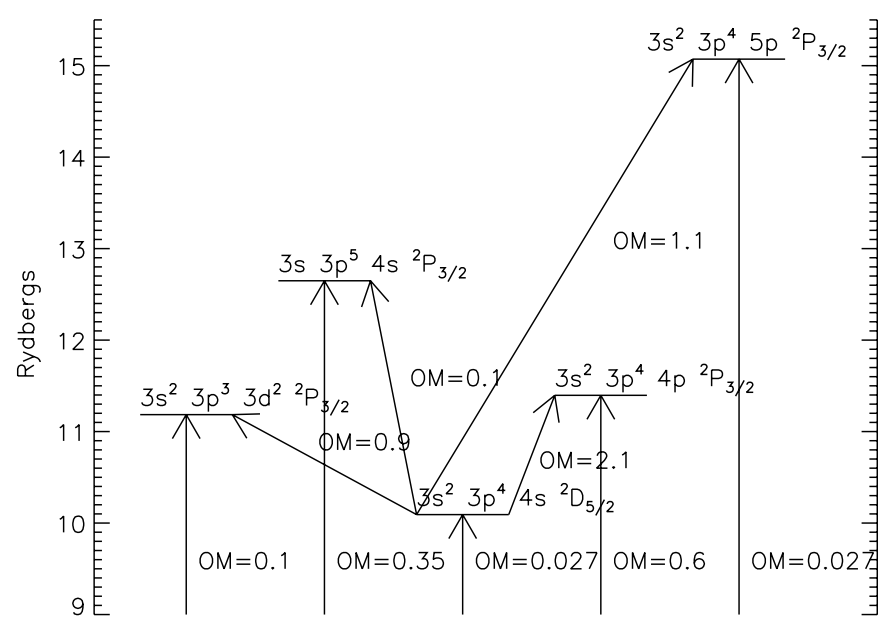

Fig. 1. The main levels related to the $3 s^{2} 3 p^{4} 4 s^{2} D_{5 / 2}$, which produces the $94.012 \AA$ line. The values of the collision strengths at threshold among the levels and from the ground state are shown.

thought of as an extrapolation of the threshold collision strength to negative final energy, this implies that the collision strength for excitation to the $3 \mathrm{~s}^{2} 3 \mathrm{p}^{4} 4 \mathrm{~s}^{2} \mathrm{D}_{5 / 2}$ level will be enhanced by 0.48 in the energy interval between the $3 \mathrm{~s}^{2} 3 \mathrm{p}^{4} 4 \mathrm{~s}^{2} \mathrm{D}_{5 / 2}$ and $3 s^{2} 3 p^{4} 4 p^{2} \mathrm{P}_{3 / 2}$ levels, almost 20 times the value for direct excitation. We will show below that the result of a detailed R-matrix treatment of the resonances gives a similar increase. These are estimates based on total threshold collision strengths. A full treatment involves detailed consideration of all the scattering channels (see, e.g. Petrini 1970).

Other contributions could also come from other configurations connected to the $3 \mathrm{~s}^{2} 3 \mathrm{p}^{4} 4 \mathrm{~s}$ by a dipole coupling, such as $3 \mathrm{~s}^{2} 3 \mathrm{p}^{3} 3 \mathrm{~d} 4 \mathrm{~s}, 3 \mathrm{~s} 3 \mathrm{p}^{5} 4 \mathrm{~s}$ and $3 \mathrm{~s}^{2} 3 \mathrm{p}^{4} n \mathrm{p}, n \geq 5$. The latter configurations are especially interesting given the large contribution from $n=4$. In practice we do not find large collision strengths for excitation of the $3 \mathrm{~s}^{2} 3 \mathrm{p}^{4} n \mathrm{p}$ for $n=5,6$ from the ground and also there are additional Auger channels reducing the branching ratio to the $3 \mathrm{~s}^{2} 3 \mathrm{p}^{4} 4 \mathrm{~s}$ levels.

We used the same approach to assess the importance of resonance contributions to the other levels of the $n=4,5$ configurations. We found that the $3 \mathrm{~s} 3 \mathrm{p}^{5} 4 \mathrm{~s}$ levels are not expected to have significant contributions from resonances. The $3 s^{2} 3 p^{4} 4 p$ levels have significant resonance contributions, mainly from the $3 s^{2} 3 p^{4} 4 d, 3 s^{2} 3 p^{4} 4 f$ and $3 s^{2} 3 p^{3} 3 d^{2}$. The $3 s^{2} 3 p^{4} 4 d$ levels are expected to have some contributions, mainly from the $3 \mathrm{~s}^{2} 3 \mathrm{p}^{4} 4 \mathrm{f}$. On the other hand, the $3 \mathrm{~s}^{2} 3 \mathrm{p}^{4}$ 4f levels are not expected to have significant resonance contributions from other $n=4,5$ levels. A similar picture applies to the $n=5$ levels.

In summary, with the exception of a small contribution from the $3 s^{2} 3 p^{4} 5 p$ levels to the $3 s^{2} 3 p^{4} 4 s$ (and $3 s^{2} 3 p^{4} 4 p$ ), the main resonance contribution to the $n=4$ spectroscopic configurations comes from configurations within the $n=4$ complex. We have therefore chosen to proceed with a full R-matrix calculation including all the main $n=3,4$ configurations.

\subsection{The R-matrix and DW calculations for the $n=3,4$ levels}

As our configuration basis set we have chosen the 32 configurations shown in Fig. 2 and listed in Table 2. The scaling parameters $\lambda_{n l}$ for the potentials in which the orbital functions are calculated are also given in Table 2. The 552 fine-structure 


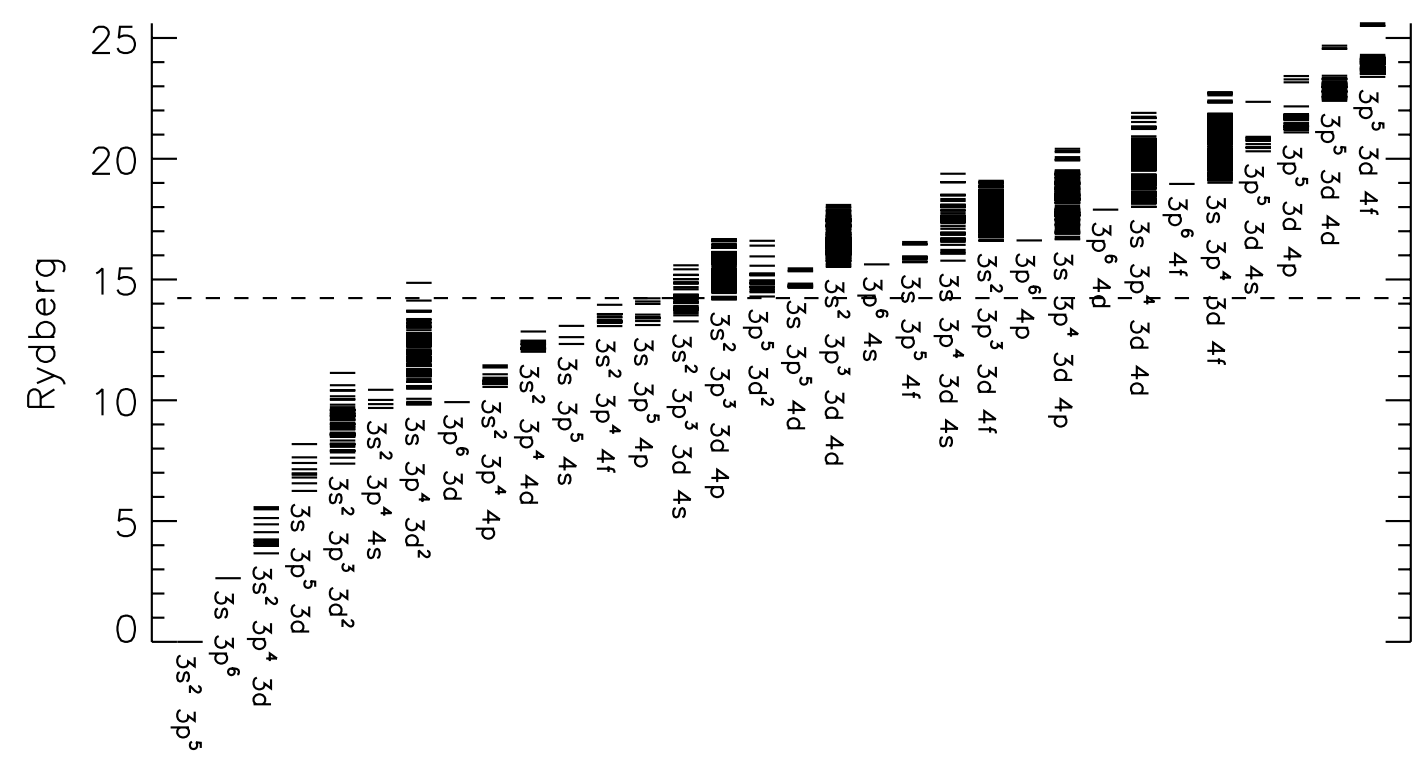

Fig. 2. The term energies of the target levels (32 configurations) for the $n=4$ calculations. The 218 terms which produce levels having energies below the dashed line have been retained for the close-coupling expansion.

Table 2. The target electron configuration basis and orbital scaling parameters $\lambda_{n l}$ for the R-matrix and DW runs for the $n=3,4$ levels.

\begin{tabular}{|c|c|c|c|}
\hline \multicolumn{3}{|c|}{ Configurations } & \multirow[b]{2}{*}{$\lambda_{n l}$} \\
\hline Even & Odd & & \\
\hline $3 s 3 p^{6}$ & $3 s^{2} 3 p^{5}$ & $1 \mathrm{~s}$ & 1.41548 \\
\hline $3 \mathrm{~s}^{2} 3 \mathrm{p}^{4} 3 \mathrm{~d}$ & $3 \mathrm{~s} 3 \mathrm{p}^{5} 3 \mathrm{~d}$ & $2 \mathrm{~s}$ & 1.12358 \\
\hline $3 s^{2} 3 p^{4} 4 s$ & $3 \mathrm{~s}^{2} 3 \mathrm{p}^{3} 3 \mathrm{~d}^{2}$ & $2 p$ & 1.06501 \\
\hline $3 s 3 p^{4} 3 d^{2}$ & $3 s^{2} 3 p^{4} 4 p$ & $3 \mathrm{~s}$ & 1.12476 \\
\hline $3 p^{6} 3 d$ & $3 s 3 p^{5} 4 s$ & $3 p$ & 1.09729 \\
\hline $3 s^{2} 3 p^{4} 4 d$ & $3 s^{2} 3 p^{4} 4 f$ & $3 d$ & 1.11252 \\
\hline $3 s 3 p^{5} 4 p$ & $3 s^{2} 3 p^{3} 3 d 4 s$ & $4 s$ & 1.21772 \\
\hline $3 s^{2} 3 p^{3} 3 d 4 p$ & $3 \mathrm{p}^{5} 3 \mathrm{~d}^{2}$ & $4 d$ & 1.20247 \\
\hline $3 p^{6} 4 s$ & $3 \mathrm{~s} 3 \mathrm{p}^{5} 4 \mathrm{~d}$ & $4 p$ & 1.19803 \\
\hline $3 s 3 p^{5} 4 f$ & $3 s^{2} 3 p^{3} 3 d 4 d$ & $4 f$ & 1.35751 \\
\hline $3 s$ 3 $p^{4} 3 d 4 s$ & $3 p^{6} 4 p$ & & \\
\hline $3 s^{2} 3 p^{3} 3 d 4 f$ & $3 s 3 p^{4} 3 d 4 p$ & & \\
\hline $3 p^{6} 4 d$ & $3 p^{6} 4 f$ & & \\
\hline $3 s 3 p^{4} 3 d 4 d$ & $3 \mathrm{~s} 3 \mathrm{p}^{4} 3 \mathrm{~d} 4 \mathrm{f}$ & & \\
\hline $3 p^{5} 3 d 4 p$ & $3 p^{5} 3 d 4 s$ & & \\
\hline $3 p^{5} 3 d 4 f$ & $3 p^{5} 3 d 4 d$ & & \\
\hline
\end{tabular}

Notes. The configurations below the line have been included in the CI expansion.

levels arising from the lowest 218 LS terms were retained for the scattering calculation. We have performed both an ICFT R-matrix and a DW calculation using the same basis. They are both large-scale calculations.

Table 3 presents a selection of fine-structure target level energies $E_{\mathrm{t}}$, compared to experimental energies $E_{\exp }$ (from Del Zanna et al. 2004, for the $n=3$ levels; otherwise from Fawcett et al. 1972). There is good overall agreement in terms of energy differences between levels. A set of "best" energies $E_{\mathrm{b}}$ was obtained with a quadratic fit between the $E_{\exp }$ and $E_{\mathrm{t}}$ values. The $E_{\mathrm{b}}$ values were used (together with the $E_{\exp }$ ones) within the R-matrix calculation to obtain an accurate position of the resonance thresholds. The resonances in the transitions to the $n=4$ levels are close to thresholds, therefore it is important to position them as accurately as possible. The $E_{\text {exp }}$ and $E_{\mathrm{b}}$ values were also used when calculating radiative rates.

The expansion of each scattered electron partial wave was done over a basis of 25 functions within the R-matrix boundary and the partial wave expansion extended to a maximum total orbital angular momentum quantum number of $L=16$. This produced accurate collision strengths up to about $80 \mathrm{Ryd}$. The resulting effective collision strengths are accurate up to an electron temperature of about $10^{7} \mathrm{~K}$. However, the interpolation for all allowed transitions utilizing the infinite limits makes the data reliable at even greater temperatures. (The collision strengths for forbidden transitions are extrapolated as $1 / E^{2}$.)

The outer region calculation includes exchange up to a total angular momentum quantum number $J=26 / 2$. We have supplemented the exchange contributions with a non-exchange calculation extending from $J=28 / 2$ to $J=74 / 2$. The outer region exchange calculation was performed in a number of stages. A coarse energy mesh was chosen above all resonances. The resonance region itself was calculated with an increasing number of energies, as was done for the Iron Project Fe XI calculation (Del Zanna et al. 2010). Pelan \& Berrington (2001) suggested that a step in energy between 0.001 and 0.002 Ryd was sufficient to resolve the resonances. Here, the number of energy points was increased from 2000 up to 8000 (equivalent to a uniform step length of $0.0018 \mathrm{Ryd}$ ). We have then considered all the transitions from the ground state and calculated the maximum deviation between the various calculations of the thermally-averaged collision strength. The results are shown in Fig. 3.

We then inspected all transitions from the ground state, and compared the collision strengths and their thermal averages with other datasets. The comparisons for a selection of levels giving rise to important transitions are displayed in Figs. 4-12. Excellent agreement between the background R-matrix and the DW collision strengths is found in all cases. This is to be expected since they both use the same target. For the main $n=3$ transitions, good overall agreement with Aggarwal \& Keenan (2005) and Del Zanna et al. (2004) is found. Interestingly, the 1-5 transition has considerably larger collision strengths than what was calculated by Del Zanna et al. (2004). The latter atomic data were used in Del Zanna (2012) where it was found that the 
Table 3. Level energies for Fe $\mathrm{x}(n=3,4)$.

\begin{tabular}{|c|c|c|c|c|c|}
\hline$\underline{l}$ & onf. & ev. & $E_{\text {exp }}$ & $E_{\mathrm{t}}$ & $E_{\mathrm{b}}$ \\
\hline 1 & $3 s^{2} 3 p^{5}$ & ${ }^{2} \mathrm{P}_{3 / 2}$ & 0.0 & 0.0 & .0 \\
\hline & & ${ }^{2} \mathrm{P}_{1 / 2}$ & 143 & $0.141(0.0$ & \\
\hline 3 & & ${ }^{2} \mathrm{~S}_{1 / 2}$ & 636 & $2.681(-0.045)$ & \\
\hline 4 & $3 s^{2} 3 p^{4} 3 d$ & ${ }^{4} \mathrm{D}_{5 / 2}$ & 542 & $3.690(-0.148)$ & $3.531(0.011)$ \\
\hline 5 & $3 s^{2} 3 p^{4} 3 d$ & ${ }^{4} \mathrm{D}_{7 / 2}$ & .542 & $3.691(-0.149)$ & $3.532(0.010)$ \\
\hline 6 & $3 s^{2} 3 p^{4} 3 d$ & ${ }^{4} \mathrm{D}_{3 / 2}$ & 3.554 & $3.700(-0.146)$ & $3.541(0.014)$ \\
\hline 7 & $3 s^{2} 3 p^{4} 3 d$ & ${ }^{4} \mathrm{D}_{1 / 2}$ & 3.568 & $3.714(-0.145)$ & 3.568 \\
\hline 8 & $3 s^{2} 3 p^{4} 3 d$ & ${ }^{4} \mathrm{~F}_{9 / 2}$ & 3.806 & $3.979(-0.1$ & $3.806(-0.000)$ \\
\hline 9 & $3 s^{2} 3 p^{4} 3 d$ & ${ }^{2} \mathrm{P}_{1 / 2}$ & - & & 3.808 \\
\hline 10 & $3 s^{2} 3 p^{4} 3 d$ & ${ }^{4} \mathrm{~F}_{7 / 2}$ & 3.853 & $4.023\left(-0.1^{\prime}\right.$ & 3.853 \\
\hline 11 & $3 s^{2} 3 p^{4} 3 d$ & ${ }^{2} \mathrm{P}_{3 / 2}$ & - & 4.054 & \\
\hline 12 & $3 s^{2} 3 p^{4} 3 d$ & ${ }^{4} \mathrm{~F}_{5 / 2}$ & 3.889 & $4.058(-$ & .889 \\
\hline 13 & $3 s^{2} 3 p^{4} 3 d$ & ${ }^{4} \mathrm{~F}_{3 / 2}$ & - & & .898 \\
\hline 14 & $3 s^{2} 3 p^{4} 3 d$ & ${ }^{4} \mathrm{P}_{1 / 2}$ & - & & 3.928 \\
\hline 15 & $3 s^{2} 3 p^{4} 3 d$ & ${ }^{2} \mathrm{D}_{3 / 2}$ & - & 4.12 & 3.944 \\
\hline 16 & $3 s^{2} 3 p^{4} 3 d$ & ${ }^{4} \mathrm{P}_{3 / 2}$ & 3.904 & 153( & $75(-0.07$ \\
\hline 17 & $3 s^{2} 3 p^{4} 3 d$ & ${ }^{4} \mathrm{P}_{5 / 2}$ & 4.014 & & 4.014 \\
\hline 18 & $3 s^{2} 3 p^{4} 3 d$ & ${ }^{2} \mathrm{~F}_{7 / 2}$ & 4.017 & & \\
\hline 19 & $3 s^{2} 3 p^{4} 3 d$ & ${ }^{2} \mathrm{D}_{5 / 2}$ & 4.035 & 4.2 & 4.035 \\
\hline 20 & $3 s^{2} 3 p^{4} 3 d$ & ${ }^{2} \mathrm{G}_{7 / 2}$ & 4.111 & & 4.111 \\
\hline & $3 s^{2} 3 p^{4} 3 d$ & ${ }^{2} \mathrm{~F}_{5 / 2}$ & 4.1 & & .123 \\
\hline 22 & $3 s^{2} 3 p^{4} 3 d$ & ${ }^{2} \mathrm{G}_{9 / 2}$ & 4.1 & & \\
\hline 23 & $3 s^{2} 3 p^{4} 3 d$ & ${ }^{2} \mathrm{~F}_{5 / 2}$ & 4.393 & & \\
\hline 24 & $3 s^{2} 3 p^{4} 3 d$ & ${ }^{2} \mathrm{~F}_{7 / 2}$ & 4.429 & 4.6 & \\
\hline 25 & $3 s^{2} 3 p^{4} 3 d$ & ${ }^{2} \mathrm{D}_{3 / 2}$ & - & & \\
\hline 26 & $3 s^{2} 3 p^{4} 3 d$ & ${ }^{2} \mathrm{D}_{5 / 2}$ & 4.704 & & \\
\hline 27 & $3 s^{2} 3 p^{4} 3 d$ & ${ }^{2} \mathrm{~S}_{1 / 2}$ & & & 38 \\
\hline 28 & $3 s^{2} 3 p^{4} 3 d$ & ${ }^{2} \mathrm{P}_{3 / 2}$ & 5.1 & & \\
\hline 29 & $3 s^{2} 3 p^{4} 3 d$ & ${ }^{2} \mathrm{P}_{1 / 2}$ & 5.193 & 5.5 & \\
\hline 30 & $3 s^{2} 3 p^{4} 3 d$ & ${ }^{2} \mathrm{D}_{5 / 2}$ & 5.221 & & 221 \\
\hline 31 & $0^{4} 3 \mathrm{~d}$ & ${ }^{2} \mathrm{D}_{3 / 2}$ & & & 5.342 \\
\hline 35 & $3 \mathrm{~s} 3 \mathrm{p}^{5} 3 \mathrm{~d}$ & ${ }^{4} \mathrm{~F}_{9 / 2}$ & 6.3 & & 63046 \\
\hline 43 & $3 s 3 p^{5} 3 d$ & ${ }^{2} \mathrm{~F}_{7 / 2}$ & & & \\
\hline 174 & $3 s^{2} 3 p^{4} 4 s$ & ${ }^{4} \mathrm{P}_{5 / 2}$ & 9.3 & & 9.308 \\
\hline 179 & $3 s^{2} 3 p^{4} 4 s$ & ${ }^{4} \mathrm{P}_{3 / 2}$ & 9.38 & & 9.383 \\
\hline 183 & ${ }^{2} 3 p^{4} 4 s$ & ${ }^{2} \mathrm{P}_{3 / 2}$ & & & 480 \\
\hline 19 & $3 s^{2} 3 p^{4} 4 s$ & ${ }^{2} \mathrm{P}_{1 / 2}$ & 9.5 & & 58 \\
\hline & $3 s^{2} 3 p^{4} 4 s$ & ${ }^{2} \mathrm{D}_{5 / 2}$ & & & $0,06 c^{2}>$ \\
\hline 203 & $3 s^{2} 3 p^{4} 4 s$ & ${ }^{2} \mathrm{D}_{3 / 2}$ & & & \\
\hline 229 & $3 s^{2} 3 p^{4} 4 p$ & ${ }^{4} \mathrm{P}_{5 / 2}$ & 10.192 & & 9) \\
\hline 243 & ${ }^{2} 3 p^{4} 4 p$ & ${ }^{4} \mathrm{D}_{7 / 2}$ & & & \\
\hline & $3 s^{2} 3 p^{4} 4 p$ & ${ }^{2} \mathrm{~F}_{5 / 2}$ & & & \\
\hline & $3 s^{2} 3 p^{4} 4 p$ & ${ }^{2} \mathrm{~F}_{7 / 2}$ & & & \\
\hline & $3 s^{2} 3 p^{4} 4 p$ & ${ }^{2} \mathrm{D}_{5 / 2}$ & & & \\
\hline 370 & $3 s^{2} 3 p^{4} 4 d$ & ${ }^{2} \mathrm{D}_{5 / 2}$ & & & \\
\hline 37 & $3 s^{2} 3 p^{4} 4 d$ & ${ }^{2} \mathrm{D}_{3 / 2}$ & & & 10) \\
\hline & $j^{4} 4 d$ & ${ }^{4} \mathrm{~F}_{5 / 2}$ & & & \\
\hline & $3 \mathrm{~s} 3 \mathrm{p}^{4} 3 \mathrm{~d}^{2}$ & ${ }^{2} \mathrm{~F}_{5 / 2}$ & & & \\
\hline & $3 s^{2} 3 p^{4} 4 d$ & ${ }^{2} \mathrm{P}_{3 / 2}$ & & & \\
\hline & $3 s^{2} 3 p^{4} 4 d$ & ${ }^{2} \mathrm{P}_{3 / 2}$ & 11.989 & 12. & \\
\hline & $3 s^{2} 3 p^{4} 4 d$ & ${ }^{2} \mathrm{P}_{1 / 2}$ & 12.005 & $20(-0$ & $11.983(0.022)$ \\
\hline & & ${ }^{2} \mathrm{D}_{5 / 2}$ & & & \\
\hline & $b^{4} 4 d$ & ${ }^{2} \mathrm{D}_{3 / 2}$ & 12.056 & & \\
\hline & $4 \mathrm{~s}$ & & - & & \\
\hline & $3 s^{2} 3 p^{4} 4 f$ & ${ }^{4} \mathrm{~F}_{9 / 2}$ & 12.652 & & \\
\hline & $3 s^{2} 3 p^{4} 4 f$ & ${ }^{4} \mathrm{G}_{11 / 2}$ & 12.732 & 13.1 & $12.732(-0.00$ \\
\hline & & ${ }^{4} \mathrm{G}_{9 / 2}$ & & $13.222(-0.465)$ & \\
\hline & $3 s^{2} 3$ & ${ }^{4} \mathrm{G}_{7 / 2}$ & & & \\
\hline & $3 s^{2} 3 p^{4} 4 f$ & & & & \\
\hline & $3 s^{2} 3 p^{4} 4 f$ & & & $13.297(-0$ & \\
\hline & $3 s^{2} 3 p^{4} 4 f$ & ${ }^{2} \mathrm{~F}_{7 / 2}$ & 12.957 & $13.385(-0.428)$ & $12.926(0.031)$ \\
\hline & & ${ }^{2} \mathrm{H}_{11 / 2}$ & 13.025 & $13.491(-0.467)$ & \\
\hline & & & & & \\
\hline & & & & $17.052(-0.000)$ & $13.559(-0.03$ \\
\hline
\end{tabular}

Notes. The experimental level energies $E_{\exp }$ (in Rydbergs, from Del Zanna et al. 2004, for the $n=3$; and Fawcett et al. 1972, for $n=4$ ) are shown, together with those obtained from our scattering target $E_{\mathrm{t}}$ and the adjusted ones, $E_{\mathrm{b}}$. Values in parentheses indicate differences with $E_{\text {exp }}$. Only a selection of levels is shown.

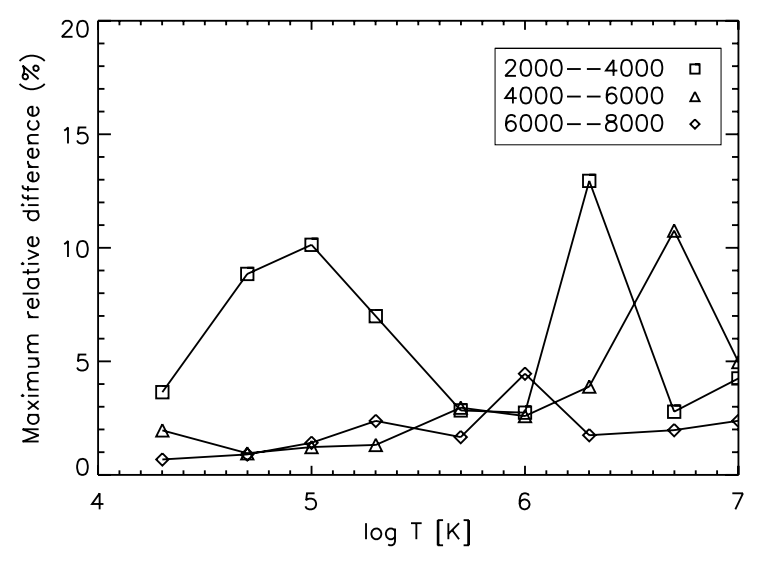

Fig. 3. Maximum relative difference (in percentage) between the thermally-averaged collision strengths from the ground state, calculated with an increasing number of energy points, from 2000 to 8000 .
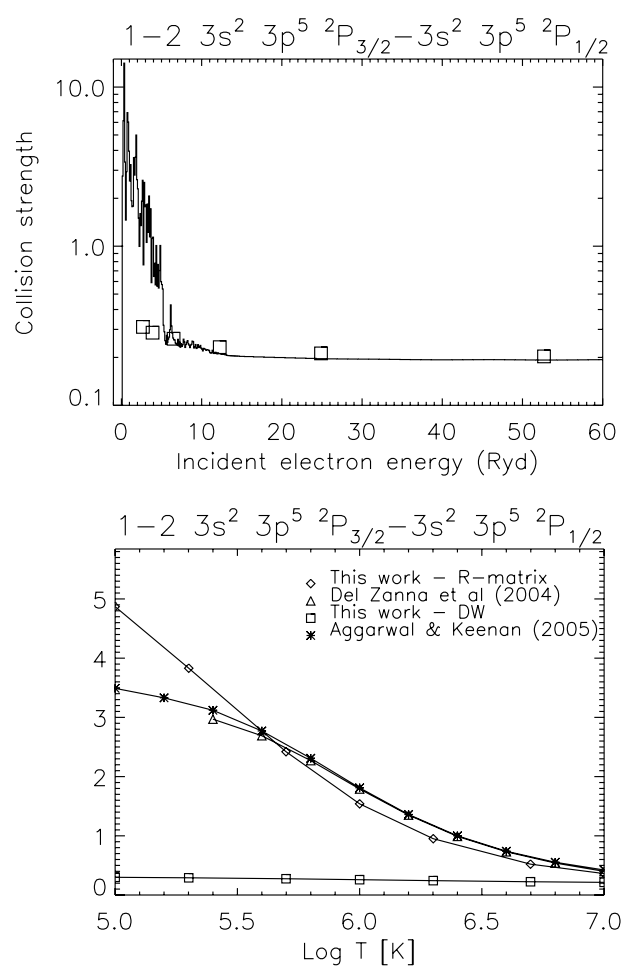

Fig. 4. Above: collision strength for the forbidden red coronal line, averaged over $0.1 \mathrm{Ryd}$ in the resonance region. The data points are displayed in histogram mode. Boxes indicate the DW values. Below: thermally-averaged collision strengths, with other calculations.

intensity of the 1-5 line was underestimated by about $50 \%$. The new atomic data remove the discrepancy.

Figure 8 shows the collision strengths for the $94.012 \AA$ transition. A strong enhancement due to resonances is present, of the same order as predicted using the approximate method of Sect. 4.2. This produces an increase of about a factor of two in the rate (and, hence, line intensities) at coronal (1 MK) temperatures. The asterisks are the Malinovsky et al. (1980) calculations, DW at 12 and 20 Ryd and semi-classical at 40 and 80 Ryd. It is clear that Malinovsky et al. (1980) overestimated the second pair of collision strengths. This produces around a factor of two increase in the rates (and, hence, line intensities) at coronal 
G. Del Zanna et al.: Atomic data for astrophysics: Fe x soft X-ray lines
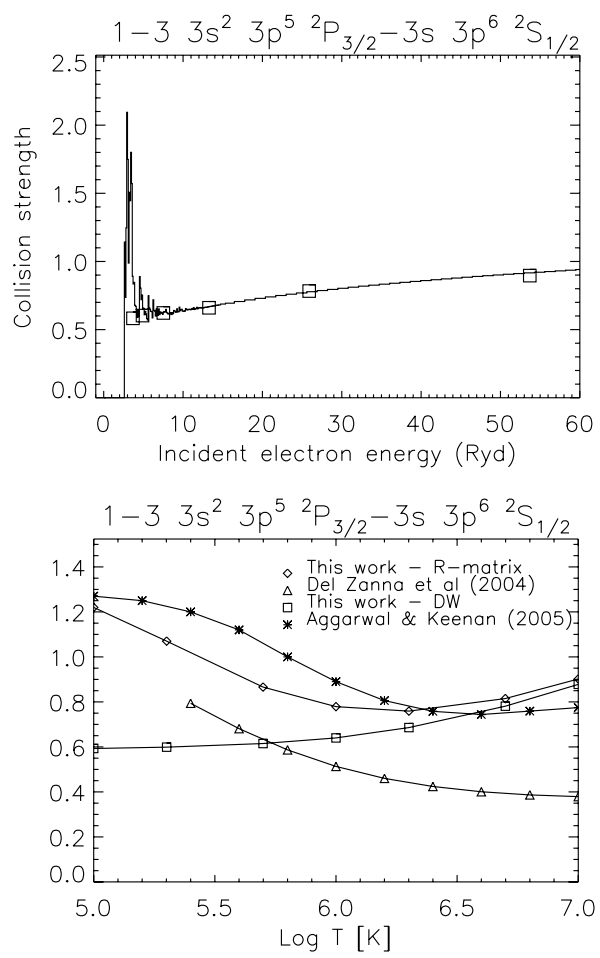

Fig. 5. Same as Fig. 4, for the allowed 1-3 $345.74 \AA$ transition.

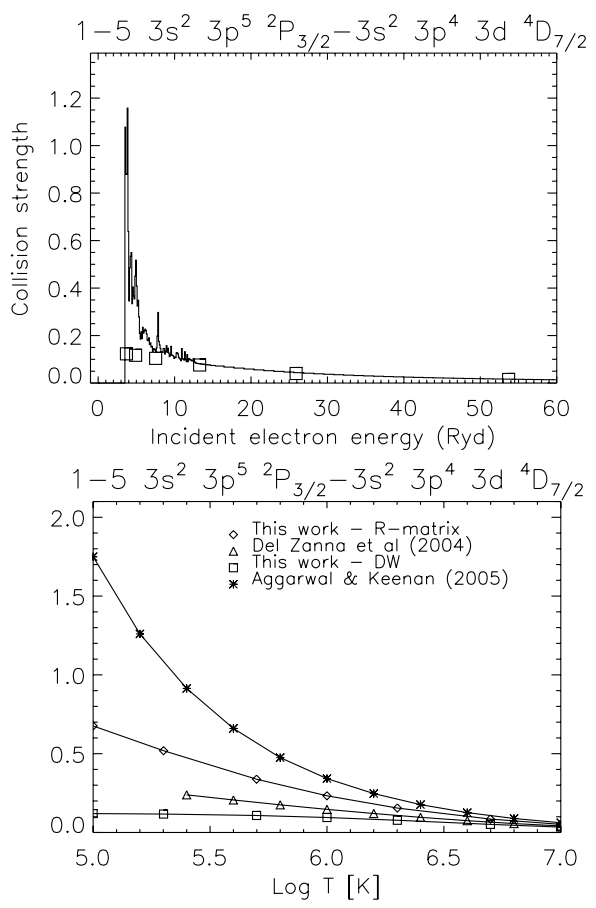

Fig. 6. Same as Fig. 4, for the strong forbidden 1-5 $257.26 \AA$ transition.

temperatures. All other lines from the same transition array show a similar behaviour.

On the other hand, the strong forbidden transition from the ground state to the $3 \mathrm{~s} 3 \mathrm{p}^{5} 4 \mathrm{~s}^{2} \mathrm{P}_{3 / 2}$ does not have much resonance contribution, as we expected, i.e. the DW and R-matrix results are almost the same, as Fig. 9 shows. The DW calculation of Malinovsky et al. (1980) is about a factor of two lower.

As we expected, we find some resonance contribution for transitions to the $3 s^{2} 3 p^{4} 4 p$ levels. Figure 10 shows an example
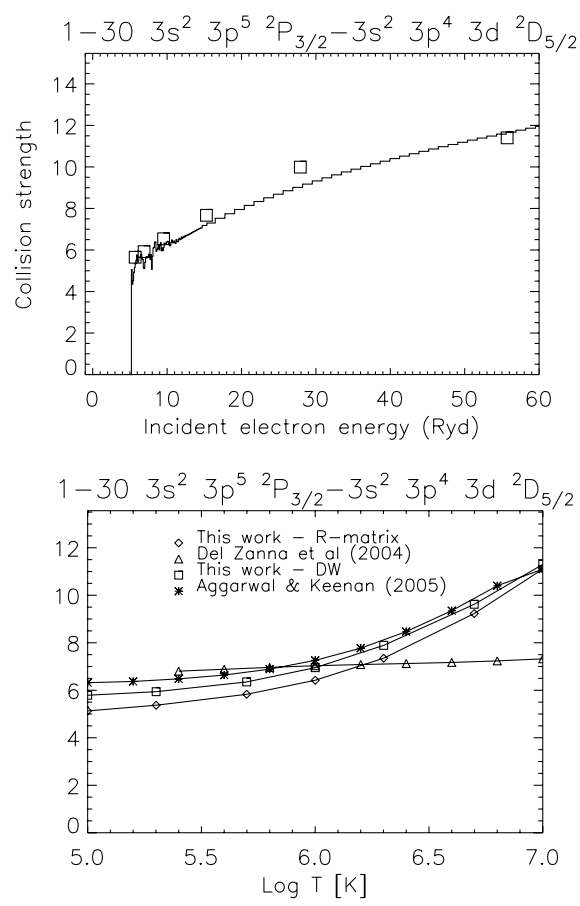

Fig. 7. Same as Fig. 4, for the allowed 1-30 174.53 $\AA$ transition, the strongest in the EUV.
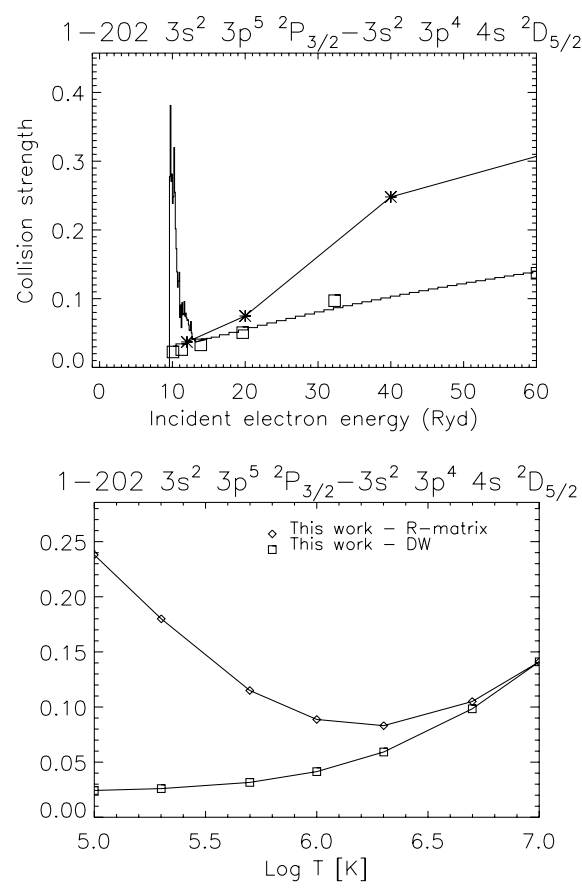

Fig. 8. Same as Fig. 4, for the allowed $94.012 \AA$ transition. Note the strong enhancement due to the resonances. The asterisks are the Malinovsky et al. (1980) calculations (DW and semi-classical).

of the excitation to a level producing one of the strongest lines (see Table 1).

An enhancement is also present in the transitions to the $3 s^{2} 3 p^{4} 4 d$ levels. Figure 11 shows one example, again for a level producing an observed line (see Table 1). Finally, Fig. 12 shows that little resonance contribution is present for the $3 s^{2} 3 p^{4} 4 f$ levels. 

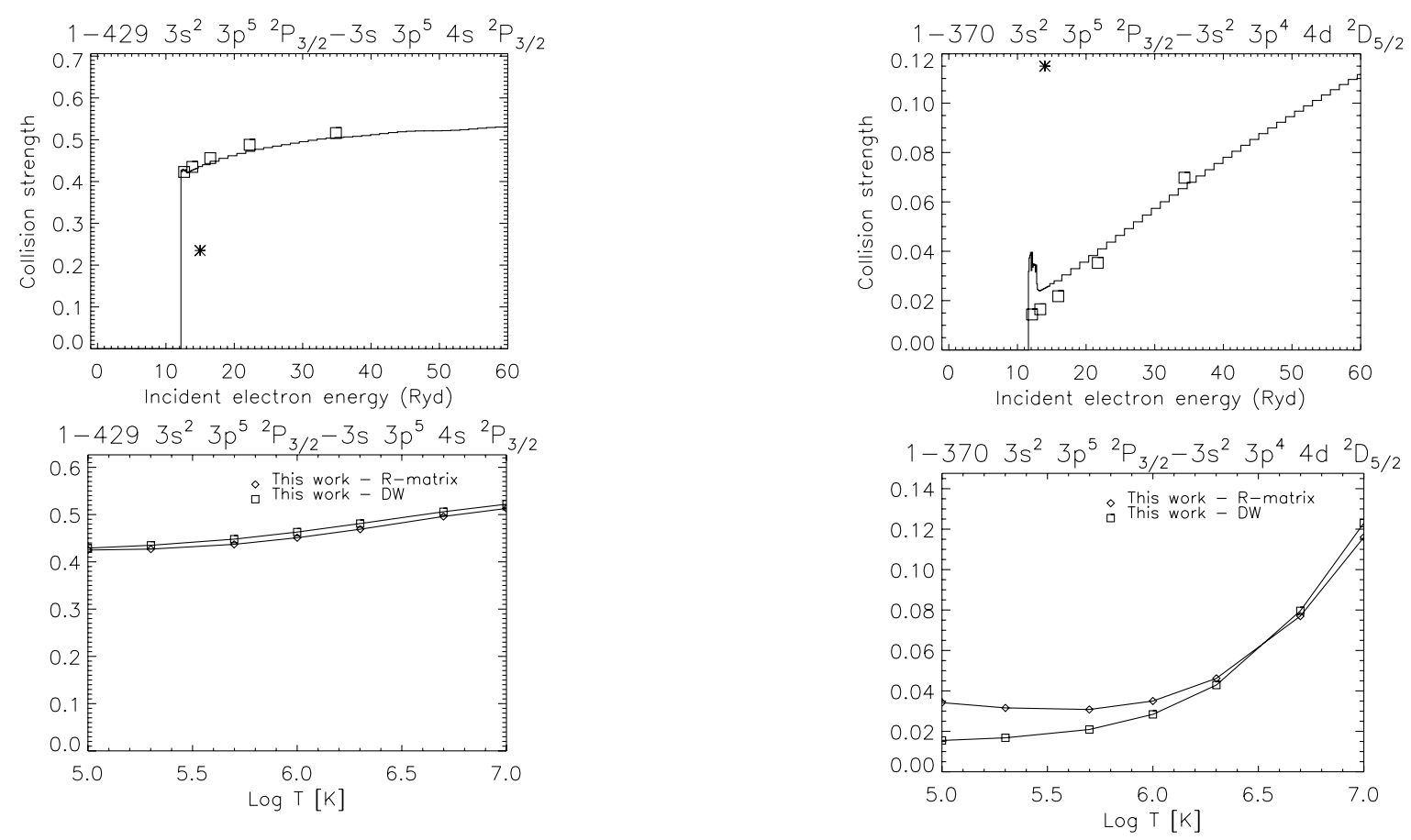

Fig. 9. Same as Fig. 4, for the strong forbidden 1-429 transition. The asterisk is the DW value calculated by Malinovsky et al. (1980).
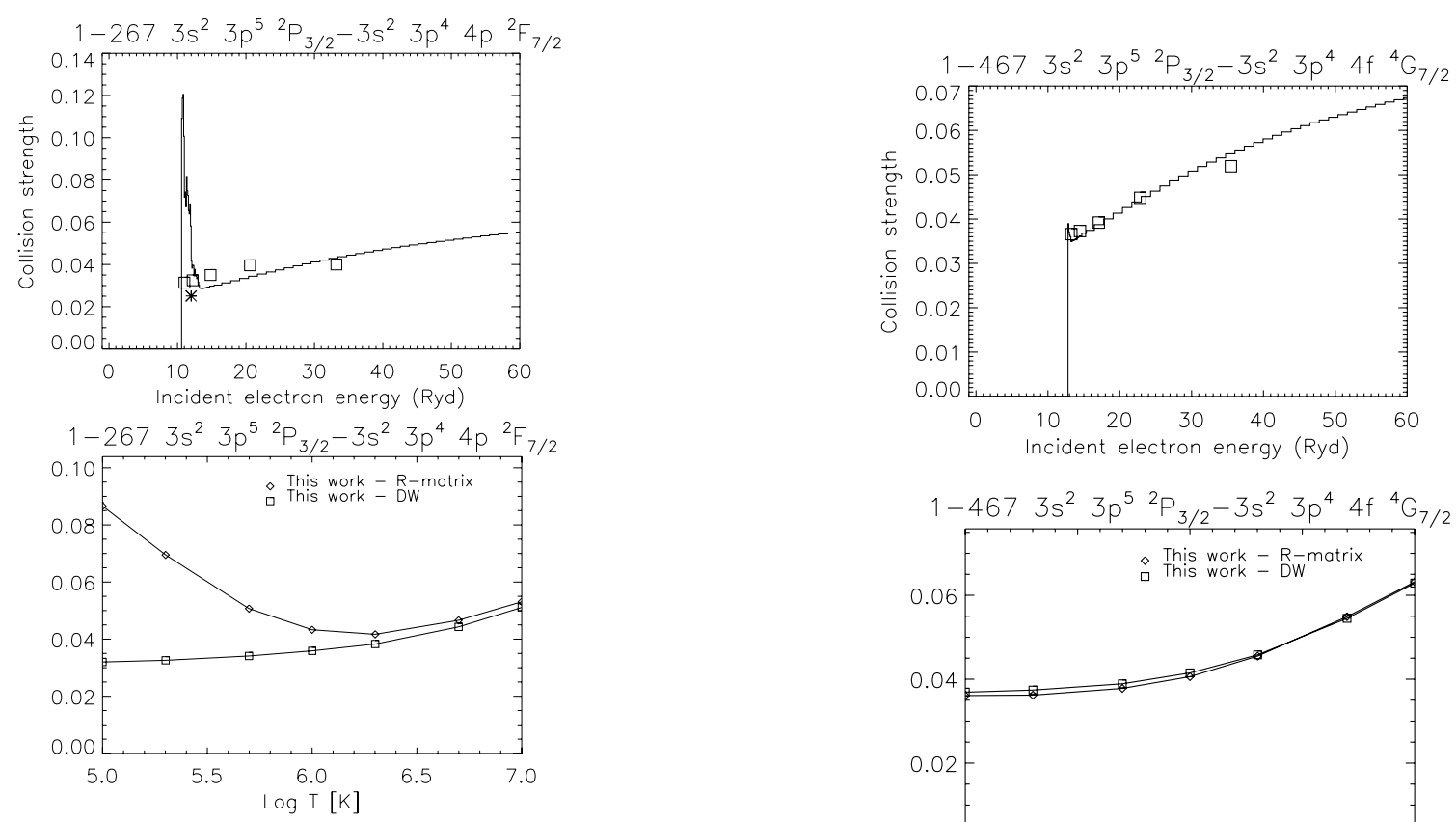

Fig. 10. Same as Fig. 4, for one of the important transitions to the $3 s^{2} 3 p^{4} 4 p$.

Fig. 11. Same as Fig. 4 , for one of the important transitions to the $3 s^{2}$
$3 p^{4} 4$. The asterisk is the DW value calculated by Malinovsky et al. (1980).

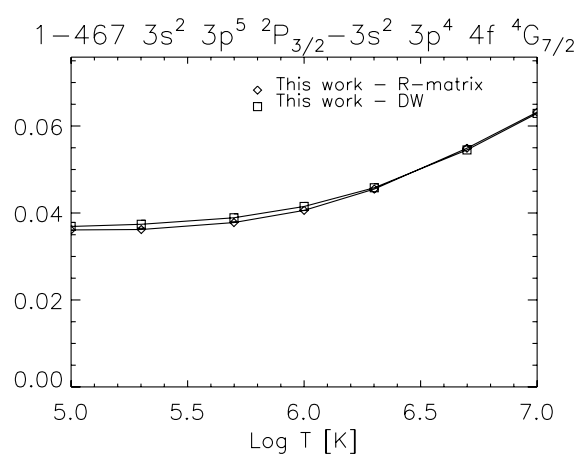

Fig. 12. Same as Fig. 4, for one of the important transitions to the $3 s^{2} 3 p^{4} 4 f$.

\subsection{Including the $n=5$, 6 levels}

We then built ion population models with the DW excitation rates and the R-matrix ones, together with the same set of radiative rates. The relative intensities are shown in the first and third intensity columns of Table 1 . The effect of the resonances is obvious. The decay from the $3 s^{2} 3 p^{4} 4 s^{2} D_{5 / 2}$ is enhanced by almost a factor of two.

In order to include the main cascading contributions from the $n=5,6$ levels in our ion model, we have run a full DW calculation (as explained in Sect. 2) with a set of 62 configurations included in the target CI expansion. We have determined collision strengths explicitly between the 1036 levels arising from the 


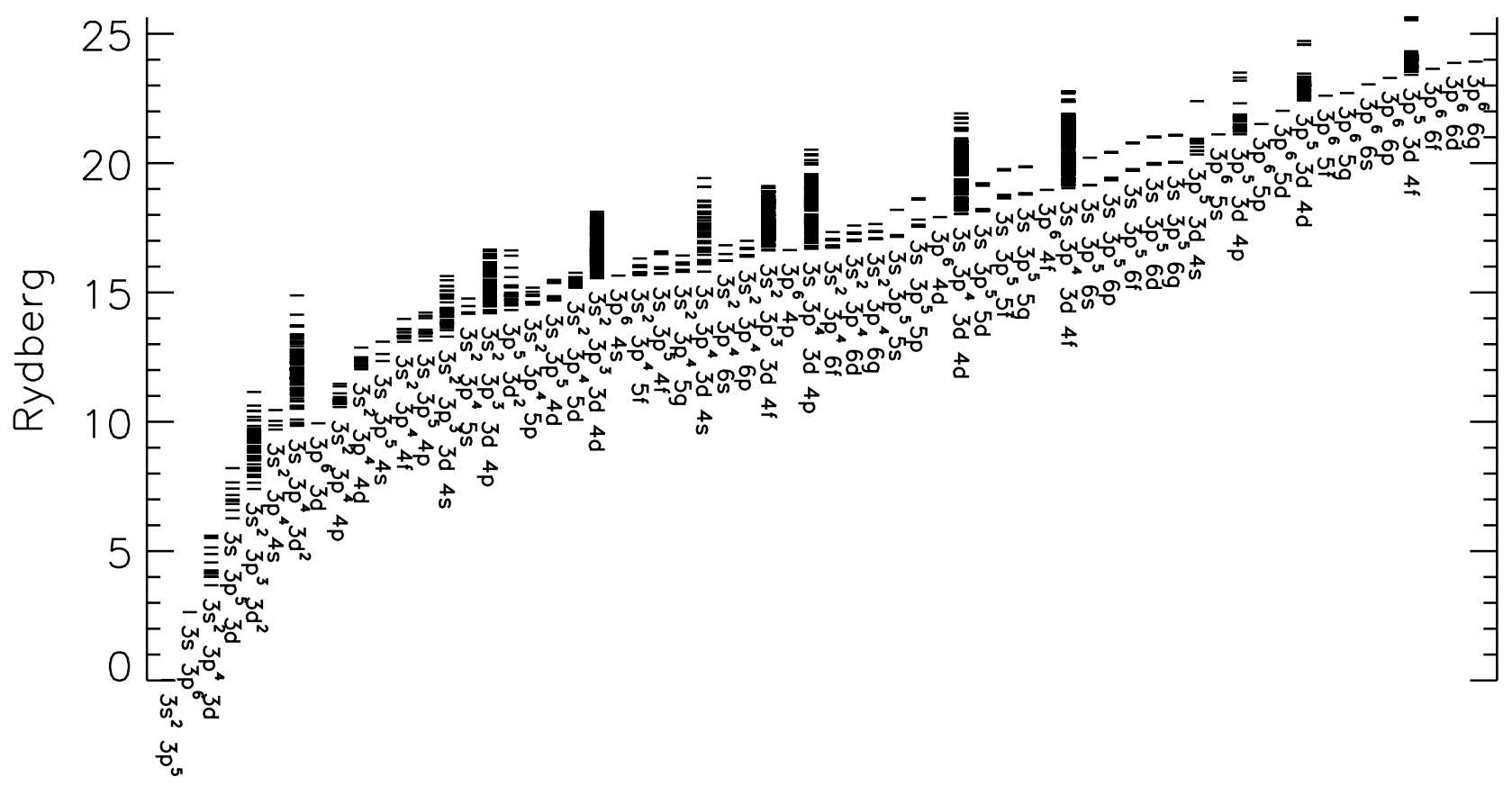

Fig. 13. The term energies of the target levels for the 62-configurations DW run (only 44 retained for the ion model).

44 configurations listed in Table 4 and displayed in Fig. 13. The excitation rates and radiative data to/from the $n=5,6$ levels have been merged with the previous R-matrix run. The level populations were obtained, and the relative intensities for the selected lines are shown in Table $1(\mathrm{RM}+\mathrm{DW}(n=6)$ model $)$. We confirm the results of Malinovsky et al. (1980), in that the inclusion of cascading from $n=5,6$ levels increases the intensities of the decays from the $3 s^{2} 3 p^{4} 4$ s levels by about $20 \%$, i.e. by a relatively small amount.

Malinovsky et al. (1980) provided some estimates of the contribution from even higher levels, and from recombination from Fe xI, however, they were even smaller.

\subsection{The 3-429 3s $3 p^{6}{ }^{2} S_{1 / 2}-3 s 3 p^{5} 4 s^{2} P_{3 / 2}$ transition}

As we have seen (cf. Fig. 9), the direct excitation from the ground to the $3 \mathrm{~s} 3 \mathrm{p}^{5} 4 \mathrm{~s}^{2} \mathrm{P}_{3 / 2}$ is large, when compared to those for the $3 \mathrm{~s}^{2} 3 \mathrm{p}^{4} 4 \mathrm{~s}$ levels. The $3 \mathrm{~s} 3 \mathrm{p}^{5} 4 \mathrm{~s}^{2} \mathrm{P}_{3 / 2}$ level decays with a strong dipole-allowed transition to the $3 \mathrm{~s} 3 \mathrm{p}^{6}{ }^{2} \mathrm{~S}_{1 / 2}(3-429)$. The resulting intensity as calculated with our most extended model is still larger than the $3 s^{2} 3 p^{5}{ }^{2} \mathrm{P}_{3 / 2}-3 s^{2} 3 p^{4} 4 s^{2} D_{5 / 2} 94.012 \AA$ line. The same is true at higher densities. It is somewhat puzzling that the main decays of the $3 s^{2} 3 p^{4} 4$ s levels were first identified by Edlén (1937), but nobody has identified the stronger 3-429 line.

We have run various DW calculations for other ions along the Cl-like sequence and for other sequences, and found the same types of transitions to be very prominent but not identified. We have found possible identifications, which will be presented in a separate paper.

For Fe $x$, the 3-429 is the only line among all the decays from the $3 s 3 p^{5} 4 s$ levels to be easily detectable. The ab-initio wavelength of the $n=4$ model is $91.5 \AA$. However, considering the relative differences between experimental and ab-initio energies of the $3 s^{2} 3 p^{4} 4 s$ and $3 s^{2} 3 p^{4} 4 p$ levels, we estimated that this line would fall around 95-96 ̊.

We have searched extensively all experimental data, in particular those B.C. Fawcett plates where transitions from the
Table 4. The target electron configuration basis and orbital scaling parameters $\lambda_{n l}$ for the 62-configurations DW run.

\begin{tabular}{|c|c|c|}
\hline Configurations & \multicolumn{2}{|r|}{$\begin{array}{r}\text { Scaling parameters } \\
\lambda_{n l}\end{array}$} \\
\hline $3 s^{2} 3 p^{5}$ & $1 \mathrm{~s}$ & 1.41548 \\
\hline $3 s^{2} 3 p^{4} 3 d$ & $2 \mathrm{~s}$ & 1.12358 \\
\hline $3 \mathrm{~s}^{2} 3 \mathrm{p}^{4} 4 l(l=\mathrm{s}, \mathrm{p}, \mathrm{d}, \mathrm{f})$ & $2 p$ & 1.06501 \\
\hline $3 \mathrm{~s}^{2} 3 \mathrm{p}^{3} 3 \mathrm{~d}^{2}$ & $3 \mathrm{~s}$ & 1.12476 \\
\hline $3 s^{2} 3 p^{3} 3 d 4 s$ & $3 p$ & 1.09729 \\
\hline $3 s 3 p^{6}$ & $3 d$ & 1.11252 \\
\hline $3 s 3 p^{5} 3 d$ & $4 \mathrm{~s}$ & 1.21772 \\
\hline $3 \mathrm{~s} 3 \mathrm{p}^{5} 4 l(l=\mathrm{s}, \mathrm{p}, \mathrm{d}, \mathrm{f})$ & $4 p$ & 1.19803 \\
\hline $3 s 3 p^{4} 3 d^{2}$ & $4 d$ & 1.20247 \\
\hline $3 \mathrm{~s} 3 \mathrm{p}^{4} 3 \mathrm{~d} 4 l(l=\mathrm{s}, \mathrm{p}, \mathrm{d}, \mathrm{f})$ & $4 f$ & 1.35751 \\
\hline $3 \mathrm{p}^{6} 3 \mathrm{~d}$ & $5 \mathrm{~s}$ & 1.17511 \\
\hline $3 \mathrm{p}^{6} 4 l(l=\mathrm{s}, \mathrm{p}, \mathrm{d}, \mathrm{f})$ & $5 \mathrm{p}$ & 1.1442 \\
\hline $3 p^{5} 3 d^{2}$ & $5 d$ & 1.16260 \\
\hline $3 \mathrm{p}^{5} 3 \mathrm{~d} 4 l(l=\mathrm{s}, \mathrm{p}, \mathrm{d}, \mathrm{f})$ & $5 f$ & 1.28045 \\
\hline $3 \mathrm{~s}^{2} 3 \mathrm{p}^{4} 5 l(l=\mathrm{s}, \mathrm{p}, \mathrm{d}, \mathrm{f}, \mathrm{g})$ & $5 \mathrm{~g}$ & 1.41348 \\
\hline $3 \mathrm{~s} 3 \mathrm{p}^{5} 5 l(l=\mathrm{s}, \mathrm{p}, \mathrm{d}, \mathrm{f}, \mathrm{g})$ & $6 s$ & 1.18612 \\
\hline $3 \mathrm{~s}^{2} 3 \mathrm{p}^{4} 6 l(l=\mathrm{s}, \mathrm{p}, \mathrm{d}, \mathrm{f}, \mathrm{g})$ & $6 \mathrm{p}$ & 1.15788 \\
\hline $3 \mathrm{~s} 3 \mathrm{p}^{5} 6 l(l=\mathrm{s}, \mathrm{p}, \mathrm{d}, \mathrm{f}, \mathrm{g})$ & $6 \mathrm{~d}$ & 1.18403 \\
\hline $3 \mathrm{p}^{6} 5 l(l=\mathrm{s}, \mathrm{p}, \mathrm{d}, \mathrm{f}, \mathrm{g})$ & $6 f$ & 1.30289 \\
\hline $3 \mathrm{p}^{6} 6 l(l=\mathrm{s}, \mathrm{p}, \mathrm{d}, \mathrm{f}, \mathrm{g})$ & $6 g$ & 1.37919 \\
\hline $3 \mathrm{~s}^{2} 3 \mathrm{p}^{3} 3 \mathrm{~d} 4 l(l=\mathrm{p}, \mathrm{d}, \mathrm{f})$ & & \\
\hline $3 \mathrm{p}^{6} 4 l(l=\mathrm{s}, \mathrm{p}, \mathrm{d}, \mathrm{f})$ & & \\
\hline $3 \mathrm{~s} 3 \mathrm{p}^{5} 4 l(l=\mathrm{d}, \mathrm{f})$ & & \\
\hline $3 \mathrm{~s} 3 \mathrm{p}^{4} 3 \mathrm{~d} 4 l(l=\mathrm{s}, \mathrm{p}, \mathrm{d}, \mathrm{f})$ & & \\
\hline $3 \mathrm{p}^{5} 3 \mathrm{~d} 4 l(l=\mathrm{s}, \mathrm{p}, \mathrm{d}, \mathrm{f})$ & & \\
\hline
\end{tabular}

Notes. The configurations below the line have been included in the CI expansion only.

$3 s^{2} 3 p^{4} 4 s$ feature prominently. We found only one candidate, an unidentified iron line at 96.01, of about the same intensity as the 
nearby 96.12 A iron line, identified by Edlén (1937). Behring et al. (1972) also observed two strong lines of the same intensity at 96.007 and $96.119 \AA$, while in all other solar measurements (e.g. Manson 1972; Malinovsky \& Heroux 1973) these lines are blended.

The only other line within a few angstroms is the $95.37 \AA$ line. This is a self-blend of two decays from the $3 \mathrm{~s}^{2} 3 \mathrm{p}^{4} 4 \mathrm{~s}$ levels, again identified by Edlén (1937). The combined intensity of these two lines at $10^{12} \mathrm{~cm}^{-3}$ is about the same as the $96.12 \AA$ one, so the $95.37 \AA$ cannot be further blended with the 3-429 transition.

We have carried out five increasingly large ab initio structure calculations just focussing on the $4 \mathrm{~s}$ configurations. The idea was to calculate the energy difference between the $3 \mathrm{~s}^{2} 3 \mathrm{p}^{4} 4 \mathrm{~s}$ ${ }^{4} \mathrm{P}_{5 / 2}$ and the $3 \mathrm{~s} 3 \mathrm{p}^{5} 4 \mathrm{~s}^{2} \mathrm{P}_{3 / 2}$ and then use the known energy of the former to estimate the wavelength of the transition from the latter state to $3 \mathrm{~s} 3 \mathrm{p}^{6}{ }^{2} \mathrm{~S}_{1 / 2}$. The results of the five calculations, in order of increasing complexity, are 91.97, 95.58, 95.78, 95.86, $95.89 \AA$ A. These are the result of purely ab initio calculations without empirical adjustments, and provide strong support for the identification of the 3-429 line with the iron $96.007 \AA$ line.

\section{Summary and conclusions}

We have presented the first complete calculations for $n=4,5,6$ levels in Fex. The calculation of accurate atomic data for the $n=4$ levels has turned out to be quite complex and for the $3 \mathrm{~s}^{2}$ $3 \mathrm{p}^{4} 4 \mathrm{~s}$, in particular, it required a large-scale R-matrix calculation. Given the small collision strengths for excitations from the ground, these levels are mainly affected in two ways. First, the rates are increased significantly by strong resonances which are attached mainly to the $3 s^{2} 3 p^{4} 4 p$ levels. Second, the population of these levels is enhanced by cascading from higher levels, as already pointed out by Malinovsky et al. (1980).

It turns out that the previous calculation for the $3 \mathrm{~s}^{2} 3 \mathrm{p}^{4} 4 \mathrm{~s}$ levels, by Malinovsky et al. (1980), overestimated the collisional rates by about a factor of two. On the other hand, we found an increase of about a factor of two due to resonances. Resonances attached to higher levels not included in the present R-matrix calculations are not expected to make a large contribution. The intensity of the $94 \AA$ as given with the $n=4$ R-matrix data and cascading from $n=4,5,6$ levels is only about $30 \%$ higher than currently calculated with CHIANTI.

We found that a large number of strong transitions are unidentified, as we saw in Fe Ix (O'Dwyer et al. 2012). We have found strong evidence in support of the identification of the $3-4293 \mathrm{~s} 3 \mathrm{p}^{6}{ }^{2} \mathrm{~S}_{1 / 2}-3 \mathrm{~s} 3 \mathrm{p}^{5} 4 \mathrm{~s}^{2} \mathrm{P}_{3 / 2}$ transition. We have found many new tentative identifications. These will be discussed in a separate paper.

The issues highlighted here are quite general in the sense that they apply to other ions along the Cl-like sequence and to other iron ions. Resonance contributions are important for many lowlying $n=4$ levels, in particular for the $3 \mathrm{~s}^{2} 3 \mathrm{p}^{q} 4 \mathrm{~s}$ levels. Decays from the $3 \mathrm{~s} 3 \mathrm{p}^{q} 4 \mathrm{~s}$ levels are strong but have not been previously identified. Work is in progress to address these issues.

Acknowledgements. G.D.Z. acknowledges the support from STFC via the Advanced Fellowship programme. We acknowledge support from STFC for the UK APAP network. B. C. Fawcett is thanked for his contribution in rescuing some of his original plates, and for the continuous encouragement over the years.

\section{References}

Aggarwal, K. M., \& Keenan, F. P. 2005, A\&A, 429, 1117

Aschwanden, M. J., \& Boerner, P. 2011, ApJ, 732, 81

Badnell, N. R. 1997, J. Phys. B At. Mol. Phys., 30, 1

Badnell, N. R. 2011, Comp. Phys. Commun., 182, 1528

Badnell, N. R., \& Griffin, D. C. 2001, J. Phys. B At. Mol. Phys., 34, 681

Behring, W. E., Cohen, L., \& Feldman, U. 1972, ApJ, 175, 493

Behring, W. E., Cohen, L., Doschek, G. A., \& Feldman, U. 1976, ApJ, 203, 521

Berrington, K. A., Eissner, W. B., \& Norrington, P. H. 1995, Comp. Phys. Comm., 92, 290

Brinkman, A. C., Gunsing, C. J. T., Kaastra, J. S., et al. 2000, ApJ, 530, L111

Burgess, A. 1964, Culham Conference on Atomic Collisions (AERE reprint 63) Burgess, A. 1965, ApJ, 141, 1588

Burgess, A. 1974, J. Phys. B At. Mol. Phys., 7, L364

Burgess, A., \& Tully, J. A. 1992, A\&A, 254, 436

Burgess, A., Chidichimo, M. C., \& Tully, J. A. 1997, J. Phys. B At. Mol. Phys., 30,33

Chidichimo, M. C., Badnell, N. R., \& Tully, J. A. 2003, A\&A, 401, 1177

Del Zanna, G. 2012, A\&A, 537, A38

Del Zanna, G., Berrington, K. A., \& Mason, H. E. 2004, A\&A, 422, 731

Del Zanna, G., Storey, P. J., \& Mason, H. E. 2010, A\&A, 514, A40

Del Zanna, G., O’Dwyer, B., \& Mason, H. E. 2011, A\&A, 535, A46

Dere, K. P., Landi, E., Mason, H. E., Monsignori Fossi, B. C., \& Young, P. R. 1997, A\&AS, 125, 149

Edlén, B. 1937, Z. Astrophys., 104, 407

Edlén, B. 1942, Z. Astrophys., 22, 30

Eissner, W. 1998, Comp. Phys. Comm., 114, 295

Eissner, W., Jones, M., \& Nussbaumer, H. 1974, Comp. Phys. Comm., 8, 270

Fawcett, B. C., Peacock, N. J., \& Cowan, R. D. 1968, J. Phys. B At. Mol. Phys., 1,295

Fawcett, B. C., Kononov, E. Y., Hayes, R. W., \& Cowan, R. D. 1972, J. Phys. B At. Mol. Phys., 5, 1255

Griffin, D. C., Badnell, N. R., \& Pindzola, M. S. 1998, J. Phys. B At. Mol. Phys., 31,3713

Grotrian, W. 1939, Naturwiss., 27, 214

Hummer, D. G., Berrington, K. A., Eissner, W., et al. 1993, A\&A, 279, 298

Landi, E., Del Zanna, G., Young, P. R., et al. 2006, ApJS, 162, 261

Lemen, J. R., Title, A. M., Akin, D. J., et al. 2011, Sol. Phys., 172

Malinovsky, L., \& Heroux, M. 1973, ApJ, 181, 1009

Malinovsky, M., Dubau, J., \& Sahal-Brechot, S. 1980, ApJ, 235, 665

Manson, J. E. 1972, Sol. Phys., 27, 107

Mason, H. E. 1975, MNRAS, 170, 651

O’Dwyer, B., Del Zanna, G., Mason, H. E., Weber, M. A., \& Tripathi, D. 2010, A\&A, 521, A21

O’Dwyer, B., Del Zanna, G., Badnell, N. R., Mason, H. E., \& Storey, P. J. 2012, A\&A, 537, A22

Pelan, J. C., \& Berrington, K. A. 2001, A\&A, 365, 258

Petrini, D. 1970, A\&A, 9, 392

Storey, P. J., Zeippen, C. J., \& Le Dourneuf, M. 2002, A\&A, 394, 753

Woods, T. N., Eparvier, F. G., Hock, R., et al. 2010, Sol. Phys., 3 